\title{
Contribuições dos Métodos de Revisão para o Desenvolvimento do Conhecimento Científico em Oncologia
}

doi: https://doi.org/10.32635/2176-9745.RBC.2019v65n4.860

Contributions of Reviews Methods for Development of Scientific Knowledge in Oncology

Contribuciones de los Métodos de Revisión para el Desarrollo del Conocimiento Científico en Oncología

Mario Jorge Sobreira da Silva'; Fernando Lopes Tavares de Lima²

Desde janeiro de 2018, a Revista Brasileira de Cancerologia (RBC) vem reestruturando sua política editorial com o intuito de ampliar o conhecimento científico em oncologia, assegurando a disseminação de manuscritos com qualidade $^{1,2}$. Neste sentido, ao longo de 2019, o Conselho Editorial da RBC optou por publicar apenas artigos de revisão que utilizem métodos "sistematizados" de pesquisa.

De forma geral, a revisão de literatura como método de pesquisa se caracteriza pela análise e síntese de um conjunto de informaçôes disponíveis sobre um determinado tema, que tem por objetivo compreender o seu estado da arte, construir teorias, desenvolver um referencial teórico-conceitual ou teórico-metodológico para a realização de pesquisas futuras, e/ou identificar aspectos que necessitam de mais investigaçáo ${ }^{3}$. Segundo Grant e Booth ${ }^{4}$, existem 14 tipos de revisóes que são mais utilizadas no contexto da saúde, porém as que empregam algum nível de sistematização metodológica têm maior potencial para serem utilizadas no contexto prático como suporte para outros estudos e para a tomada de decisão baseada em evidência.

Enquanto as revisôes "não sistematizadas" apresentam perguntas de pesquisas amplas, frequentemente não especificam as fontes de informação (o que pode gerar algum viés de seleção) e possuem técnicas de análises variadas; as revisōes "sistematizadas" apresentam perguntas de pesquisa específicas, têm fontes de informação definidas e parâmetros de seleção claros (permitindo a sua reprodutibilidade) e utilizam técnicas de análises criteriosas ${ }^{5}$. Os métodos de revisão "sistematizados", que têm sido mais difundidos na área da oncologia nos últimos anos, são: as revisôes sistemáticas com ou sem metanálise, as integrativas, as de escopo (scoping review) e as metassínteses qualitativas.

A revisão sistemática é considerada o tipo de revisão mais conhecido 5 . Se caracteriza por buscar sintetizar todo o conhecimento disponível sobre um determinado tema, geralmente com base em informaçóes oriundas de estudos experimentais, principalmente ensaios clínicos randomizados, e a partir do uso de técnicas rigorosas de seleção e de análise com o intuito de evitar vieses e de fornecer evidências confiáveis e significativas que suportem a tomada de decisão em saúde e o desenvolvimento de diretrizes clínicas e terapêuticas ${ }^{6}$. Quando acompanhadas de metanálises, essas revisōes empregam critérios específicos de extração de dados e técnicas estatísticas que permitam a agregação de dados quantitativos de diferentes estudos semelhantes, levando em consideração o tamanho amostral de cada estudo ${ }^{3}$. Independente do estudo empregar ou não metanálise, é fundamental que exista um protocolo de pesquisa devidamente registrado e publicado antes do seu início ${ }^{7}$. Uma base internacional possível de ser utilizada para essa finalidade é o PROSPERO (https://www.crd.york.ac.uk/prospero/). Além disso, no momento de relatar os resultados da pesquisa, é recomendado que os pesquisadores utilizem, para revisōes de estudos experimentais, a recomendação PRISMA - Preferred Reporting Items for Systematic Reviews and Meta-Analyses ${ }^{8}$ e, para revisôes de estudos epidemiológicos observacionais, o checklist Moose - Meta-analysis of Observational Studies in Epidemiology, ${ }^{9}$.

As revisôes integrativas são um tipo específico de revisão que sintetiza informaçóes provenientes de estudos empíricos ou teóricos, com o intuito de fornecer uma compreensão mais ampliada de um determinado fenômeno ou problema de saúde ${ }^{11}$. A manutenção da integridade científica e de técnicas rigorosas e criteriosas de seleção e análise durante a realização desse tipo de revisão é fundamental, de maneira que o resultado possa contribuir de forma significativa para a prática clínica e para a realização de outras pesquisas ${ }^{12}$. O processo de revisão integrativa é realizado em cinco etapas: (1) identificação do problema e da pergunta específica de pesquisa; (2) definição das bases de dados que

${ }^{2}$ Coordenação de Ensino do INCA. E-mail:flima@inca.gov.br. Rio de Janeiro (RJ), Brasil. ORCID: https://orcid.org/0000-0002-8618-7608 
serão pesquisadas, dos critérios de seleção, considerando a pergunta norteadora; (3) avaliação dos dados, focada na autenticidade, qualidade, valor informacional e representatividade. Para tanto, é importante que a coleta dos dados tenha sido realizada de maneira precisa, por meio do uso de instrumentos apropriados; (4) análise crítica, que permita realizar a síntese adequada dos dados, assim como sua exibição, discussão e conclusôes; e (5) a apresentação da revisão, caracterizada pela síntese clara e abrangente, retratando o processo de integração e descrevendo informações pertinentes e detalhadas, além das limitaçóes da pesquisa ${ }^{13,14}$.

Por sua vez, as revisóes do tipo scoping review representam uma metodologia que apresenta os seguintes propósitos: avaliar evidências emergentes; esclarecer conceitos ou definiçôes; analisar como estão sendo conduzidas pesquisas em um determinado campo do conhecimento; identificar fatores relacionados a um determinado tema; subsidiar a realização de revisôes sistemáticas; e identificar e analisar lacunas do conhecimento científico ${ }^{6}$. Em particular, esse tipo de revisão é utilizado quando não é possível a realização de uma revisão sistemática, embora uma diversidade de critérios metodológicos tenha que ser rigorosamente empregada na realização da pesquisa ${ }^{15}$. No sentido de garantir a qualidade metodológica e relatos adequados desse tipo de estudo, é recomendado que os pesquisadores utilizem o checklist PRISMA Extension for Scoping Reviews (PRISMA-ScR) ${ }^{16}$.

Já as metassínteses qualitativas visam a fornecer uma vasta gama de significados, experiências e perspectivas dos participantes nos contextos de saúde. Essas pesquisas podem reunir dados em diferentes contextos, gerar novos modelos teóricos ou conceituais, identificar lacunas de pesquisa, informar o desenvolvimento de estudos primários e fornecer evidências para o desenvolvimento, implementação e avaliação de intervenções em saúde ${ }^{17}$. Muitos aspectos dos métodos para sintetizar a pesquisa qualitativa estão nos estágios iniciais de desenvolvimento e o protocolo ENTREQ - Enhancing transparency in reporting the synthesis of qualitative research ${ }^{17}$ tem sido utilizado como guia para os autores.

A escolha do tipo de revisão depende dos objetivos do autor e do objeto de estudo. Uma vez tendo definido o modelo de revisão "sistematizada", é fundamental que os autores sigam os parâmetros definidos na literatura, buscando assim a desejada qualidade no relato científico.

Desejamos que o aprimoramento das políticas editoriais da RBC possa contribuir para a publicação de pesquisas que colaborem para a melhoria da qualidade das estratégias de controle do câncer. Esperamos, ainda, que as orientaçôes fornecidas neste editorial possam fomentar a submissão de estudos com o uso de revisôes "sistematizadas" para a RBC nos próximos anos.

\section{REFERÊNCIAS}

1. Bergmann A, Casado L, Siqueira ASE, et al. Reestruturando uma revista científica do SUS com base na agenda 2030. Rev Bras Cancerol. 2018;64(1):7-8. doi: https://doi.org/10.32635/2176-9745.RBC.2018v64n1.114

2. Siqueira ASE, Casado L, Bergmann A, et al. A disseminação de conhecimento científico e a qualidade da informação. Rev Bras Cancerol. 2019;65(1):e-0029. doi: https://doi.org/10.32635/2176-9745.RBC.2019v65n1.291

3. Paré G, Trudel MC, Jaana M, et al. Synthesizing information systems knowledge: a typology of literature reviews. Inf Manag. 2015;52(2):183-99. doi: https://doi.org/10.1016/j.im.2014.08.008

4. Grant MJ, Booth A. A typology of reviews: an analysis of 14 review types and associated methodologies. Health Info Libr J. 2009;26(2):91-108. doi: https://doi.org/10.1111/j.1471-1842.2009.00848.x

5. Botelho LLR, Cunha CCA, Macedo M. O método da revisão integrativa nos estudos organizacionais. Gest Soc. 2011;5(11):121-36. doi: https://doi.org/10.21171/ges.v5i11.1220

6. Munn Z, Peters MDJ, Stern C. et al. Systematic review or scoping review? Guidance for authors when choosing between a systematic or scoping review approach. BMC Med Res Methodol. 2018;18(1):143. doi: https://doi.org/10.1186/ s12874-018-0611-x

7. Silagy CA, Middleton P, Hopewell S. Publishing protocols of systematic reviews: comparing what was done to what was planned. Jama. 2002;287(21):2831-4. doi: https://doi.org/10.1001/jama.287.21.2831

8. Moher D, Liberati A, Tetzlaff J, et al. Preferred reporting items for systematic reviews and meta-analyses: the PRISMA statement. PLoS Med. 2009;6(7):e1000097. doi: https://doi.org/10.1371/journal.pmed.1000097

9. Ministério da Saúde (BR), Secretaria de Ciência, Tecnologia e Insumos Estratégicos, Departamento de Ciência e Tecnologia. Diretrizes metodológicas: elaboração de revisão sistemática e metanálise de estudos observacionais comparativos sobre fatores de risco e prognóstico. Brasília: Ministério da Saúde; 2014.

10. Stroup DF, Berlin JA, Morton SC, et al. Meta-analysis of observational studies in epidemiology: a proposal for reporting. Meta-analysis of Observational Studies in Epidemiology (MOOSE) group. JAMA. 2000;283(15):2008-12. doi: https:// doi.org/10.1001/jama.283.15.2008 
11. Whittemore R, Knafl K. The integrative review: updated methodology. J Adv Nurs. 2005;52(5):546-53. doi: https:// doi.org/10.1111/j.1365-2648.2005.03621.x

12. Russell CL. An overview of the integrative research review. Prog Transplant. 2005;15(1):8-13. doi: https://doi. org/10.7182/prtr.15.1.0n13660r26g725kj

13. Hopia H, Latvala E, Liimatainen L. Reviewing the methodology of an integrative review. Scand J Caring Sc. 2016;30(4):662-9. doi: https://doi.org/10.1111/scs.12327

14. Souza MT, Silva MD, Carvalho R. Revisão integrativa: o que é e como fazer. Einstein (São Paulo). 2010;8(1):102-6. doi: http://dx.doi.org/10.1590/s1679-45082010rw1134

15. Peters MD, Godfrey CM, Khalil H, et al. Guidance for conducting systematic scoping reviews. Int J Evid Based Health. 2015;13(3):141-6. doi: https://doi.org/10.1097/XEB.0000000000000050

16. Tricco AC, Lillie E, Zarin W, et al. PRISMA extension for scoping reviews (PRISMA-ScR): checklist and explanation. Ann Intern Med. 2018;169(7):467-73. doi: https://doi.org/10.7326/M18-0850

17. Tong A, Flemming K, McInnes E, et al. Enhancing transparency in reporting the synthesis of qualitative research: ENTREQ. BMC Med Res Methodol. 2012;12:181. doi: https://doi.org/10.1186/1471-2288-12-181 